\title{
Betulinic acid impairs metastasis and reduces immunosuppressive cells in breast cancer models
}

\author{
An-Qi Zeng ${ }^{1, *}$, Yan Yu ${ }^{1, *}$, Yu-Qin Yao, ${ }^{1, *}$, Fang-Fang Yang ${ }^{1}$, Mengya Liao ${ }^{2}$, Lin-Jiang \\ Song ${ }^{1}$, Ya-Li Li ${ }^{1}$, Yang $\mathbf{Y u}^{3}$, Yu-Jue $\mathrm{Li}^{1}$, Yuan-Le Deng ${ }^{1}$, Shu-Ping Yang ${ }^{1}$, Chen-Juan \\ Zeng $^{1,4}$, Ping Liu ${ }^{5}$, Yong-Mei Xie ${ }^{1}$, Jin-Liang Yang ${ }^{1}$, Yi-Wen Zhang ${ }^{1}$, Ting-Hong Ye $^{1}$ \\ and Yu-Quan Wei ${ }^{1}$ \\ ${ }^{1}$ Laboratory of Liver Surgery, State Key Laboratory of Biotherapy, West China Hospital, Sichuan University and Collaborative \\ Innovation Center for Biotherapy, Chengdu 610041, China \\ ${ }^{2}$ Sichuan Nursing Vocational College, Chengdu 610100, China \\ ${ }^{3}$ Department of Peritoneal Cancer Surgery, Beijing Shijitan Hospital Affiliated to the Capital Medical University, Beijing \\ 100038, China \\ ${ }^{4}$ Sichuan Scientist Biotechnology Co., Ltd, Chengdu 610041, China \\ ${ }^{5}$ Department of Gynecology and Obstetrics, Key Laboratory of Birth Defects and Related Diseases of Women and Children of \\ the Ministry of Education, West China Second Hospital, Sichuan University, Chengdu 610041, China \\ *These authors contributed equally to this work \\ Correspondence to: Ting-Hong Ye, email: yeth1309@scu.edu.cn \\ Yi-Wen Zhang, email: yiwenzhang@scu.edu.cn \\ Keywords: betulinic acid; breast cancer; migration and invasion; pulmonary metastases; MDSCs
}

Received: May 19, $2017 \quad$ Accepted: November 27, $2017 \quad$ Published: December 17, 2017

Copyright: Zeng et al. This is an open-access article distributed under the terms of the Creative Commons Attribution License 3.0 (CC BY 3.0), which permits unrestricted use, distribution, and reproduction in any medium, provided the original author and source are credited.

\section{ABSTRACT}

Breast cancer is the most common female cancer with considerable metastatic potential, explaining the need for new candidates that inhibit tumor metastasis. In our study, betulinic acid (BA), a kind of pentacyclic triterpenoid compound derived from birch trees, was evaluated for its anti-metastasis activity in vitro and in vivo. BA decreased the viability of three breast cancer cell lines and markedly impaired cell migration and invasion. In addition, BA could inhibit the activation of stat 3 and FAK which resulted in a reduction of matrix metalloproteinases (MMPs), and increase of the MMPs inhibitor (TIMP-2) expression. Moreover, in our animal experiment, intraperitoneal administration of $10 \mathrm{mg} / \mathrm{kg} /$ day BA suppressed 4T1 tumor growth and blocked formation of pulmonary metastases without obvious side effects. Furthermore, histological and immunohistochemical analyses showed a decrease in MMP-9 positive cells, MMP-2 positive cells and Ki-67 positive cells and an increase in cleaved caspase- 3 positive cells upon BA administration. Notably, BA reduced the number of myeloid-derived suppressor cells (MDSCs) in the lungs and tumors. Interestingly, in our caudal vein model, BA also obviously suppressed 4T1 tumor pulmonary metastases. These findings suggested that BA might be a potential agent for inhibiting the growth and metastasis of breast cancer.

\section{INTRODUCTION}

According to statistics, the three most commonly diagnosed types of cancer in women were breast, lung and colorectum in the USA in 2015. In particular, breast cancer, alone is expected to account for approximately
$29 \%$ of all yearly diagnosed cancer cases in women [1]. Moreover, the occurrence of breast cancer-like most cancer is step up in developing countries such as Brazil and China [2]. Although progresses have been made in breast cancer, the huge threat of this cancer for women is still serious, and particularly the patients who suffer from the "triple- 
negative' breast cancer (TNBC), which is negative for the expressions of progesterone (PR), estrogen receptors (ER) and HER2 [3-5]. The terminal TNBC have an aggressive clinical progress with a bad prognosis compared with non-TNBC [6]. Besides, highly malignant and metastatic of breast cancer is a principle cause of female mortality [7]. Unfortunately, there is no effective therapy to control the reappearance and metastasis of breast cancer. Thus, finding potent and effective candidates for metastatic breast cancer with potential anti-tumor activity and low toxicity is necessary.

Natural products have been used to treat human diseases for thousands of years and have precious values in drug discovery and development $[8,9]$. Most of anticancer and anti-infectious agents are derived from natural products [9]. Furthermore, in recent decades, the rapid development of more effective drugs with fewer, lesssevere side effects is a common goal shared by scientists and clinicians [10]. Therefore, the identification of the naturally occurring phytocompounds from medicinal plants and natural products to combat diseases has become crucial due to its low side effects [11]. Betulinic acid (BA) (Supplementary Figure 1) (3ß, hydroxyl-lup-20(29)-en-28oic acid) is a natural pentacyclic triterpene with a lupine structure, which is found in plant sources including various bark extractant, acuminatissima leaves, and wild jujube seeds $[8,12,13]$. Studies have demonstrated that BA has a variety of beneficial biological activities, including anti-cancer, anti-viral, anti-bacterial, anti-malarial and anti-inflammatory activity [14-16]. Moreover, BA could suppress tumorigenesis in various types of cancer, including breast, lung, colon, pancreatic, and prostate cancer [17-21].

As to breast cancer, it is reported that BA can inhibit viability of breast cancer cells and angiogenesis, and induce mitochondrial pathway of cell apoptosis $[17,22]$. The antitumor mechanism of BA in breast cancer cells have been reported, at least including topoisomerase [23], transcription factor nuclear factor kappa B (NF-kB) [24], vascular endothelial growthfactor (VEGF) and its receptor (VEGFR) [25], and multidrug resistance proteins [26]. However, the effects of BA on suppressing pulmonary metastasis of breast cancer and its related molecular mechanism have not yet been reported. Considering the effects of BA on breast cancer, we hypothesized that BA might inhibit the metastasis of breast cancer. Therefore, the anti-proliferation and anti-metastasis efficacy of BA in vitro and in vivo were assessed in the current study. Furthermore, the antimetastasis mechanism of BA were also explored.

\section{RESULTS}

\section{BA inhibits breast cancer cells proliferation}

To evaluate whether BA has direct effects on breast cancer cells, we treated three established breast cancer cell lines with BA and detecting cell viability by $3-(4,5)$-dimethylthiahiazo(-z-y1)-3,5-diphenytetrazoliumromide(MTT). Treatment of MCF-7, 4T1 and MDA-MB-231 cells with various concentration of BA for 48 and $72 \mathrm{~h}$ respectively, resulted in a concentration-dependent decrease in the cell viability (Figure 1A). These data suggested that BA could inhibit breast cancer cells viability in a concentration and timedependent manner. To further examine whether BA could inhibit proliferation of breast cancer cells, we conducted clonogenic assay to visually assess the anti-proliferation activity of BA. As shown in Figure 1B, clonogenic assay definitely showed that clone formation of 4T1, MCF-7 and MDA-MB-231 cells was significantly reduced in a concentration-dependent manner after exposure to BA. Meanwhile, the size of the colonies treated with BA was significantly smaller than the control group. These results were consistent with the MTT data. Taken together, those resultsconfirmed that $\mathrm{BA}$ had a strong cytostatic and cytotoxic effect on breast cancer cells.

\section{BA inhibits breast cancer cell migration and invasion}

Migration and invasion of tumor cells are regarded as a crucial step for initial breast cancer metastasis [27, 28]. Therefore, it is necessary to investigate whether BA could inhibit breast cancer cell migration and invasion. To test the effects of BA on migration, we performed wound-healing and transwell migration assays using 4T1 and MDA-MB-231 cell lines. As shown in Figure 2A, BA inhibited migration of both 4T1 and MDA-MB-231 cells in dose-dependent manners. Similar results were obtained in transwell migration assays (Figure 2B and Supplementary Figure 2). Furthermore, we made a Matrigel invasion assay. Figure $2 \mathrm{C}$ showed that both 4T1 and MDA-MB-231 cells exhibit significantly decreased invasion in the presence of BA than control groups. Matrix metalloproteinases (MMPs) have been identified as the major molecules in cancer metastasis [27]. Stat3 and $\mathrm{Src} / \mathrm{FAK} / \mathrm{Rac} 1$ signal are known as an vital role in controlling cell migration and invasion by regulating the expression level of genes included MMP2, 9 and it is established that the level of MMPs is positively related to cancer cell metastasis. [28, 29] Therefore, we also investigated whether MMP-2, MMP-9 and TIMP-2 are considered to be related with cell migration and invasion by BA. As Figure 2D, 2E and Supplementary Figure 3 indicated, BA treatment decreased the expression of MMP-2 and MMP-9 while increased the expression of TIMP-2 in 4T1 and MDA-MB-231 cells. Moreover, The results of western blot indicated that the treatment with BA significantly inhibited the levels of p-Stat3 and P-FAK in 4T1 and MDA-MB-231 cells(Figures 2D, 2E and Supplementary Figure 3). Altogether, all of these results indicated that BA possessed a strong ability on breast cancer cell migration and invasion. 
A

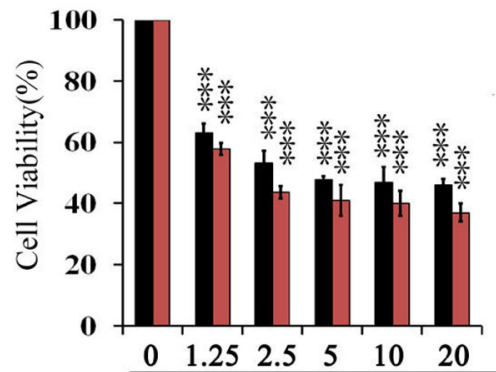

$4 \mathrm{~T} 1$

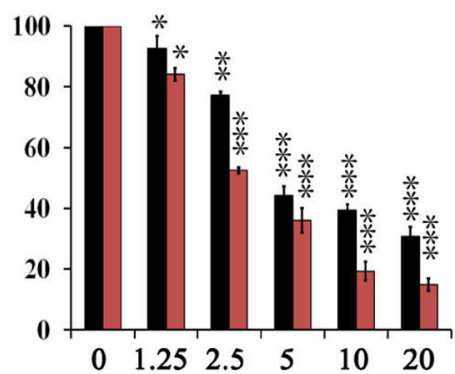

MDA-MB-231

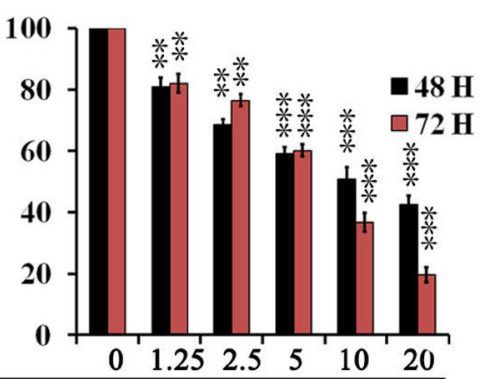

B
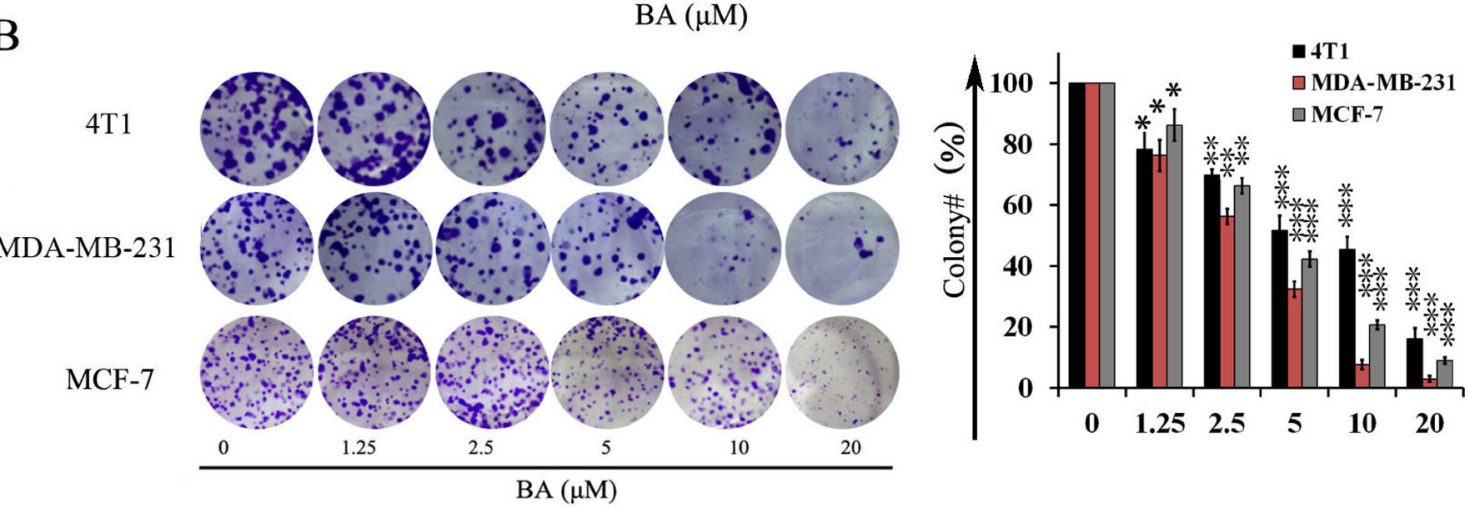

Figure 1: The effects of BA in breast cancer cells viability. (A) Proliferation of MCF-7, 4T1 and MDA-MB-231 cells treated with various concentrations $(0-20 \mu \mathrm{M})$ of BA for $48 \mathrm{~h}$ and $72 \mathrm{~h}$. Cell viability was evaluated by MTT assay. Data represent mean \pm SD at least from 3 independent experiments. (B) The effects of BA $(0-20 \mu \mathrm{M})$ on colony formation in 4T1 and MDA-MB-231 cell lines for 12 days, the statistic results of colony formation assays presented surviving colonies. Data are expressed as mean \pm SD at least from 3 independent experiments $\left({ }^{*} P<0.05 ;{ }^{* *} P<0.01 ;{ }^{* * *} P<0.001\right)$.

A
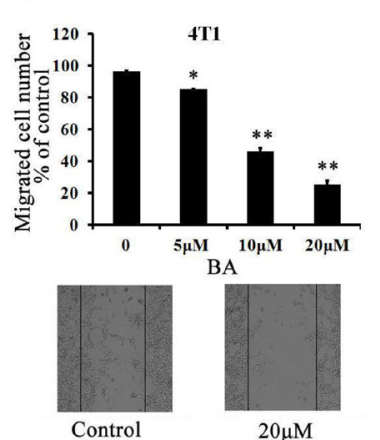

C

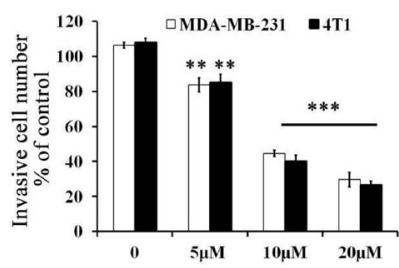

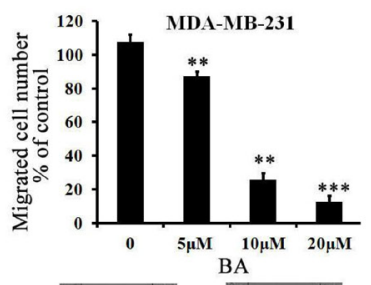

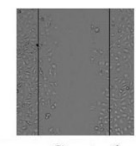

Control

D

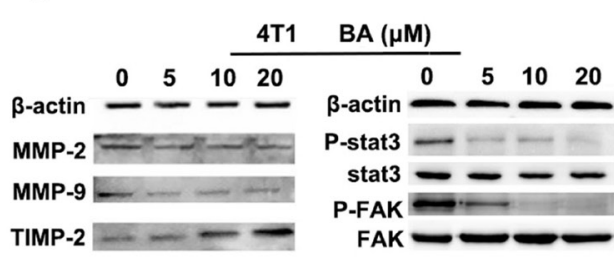

B
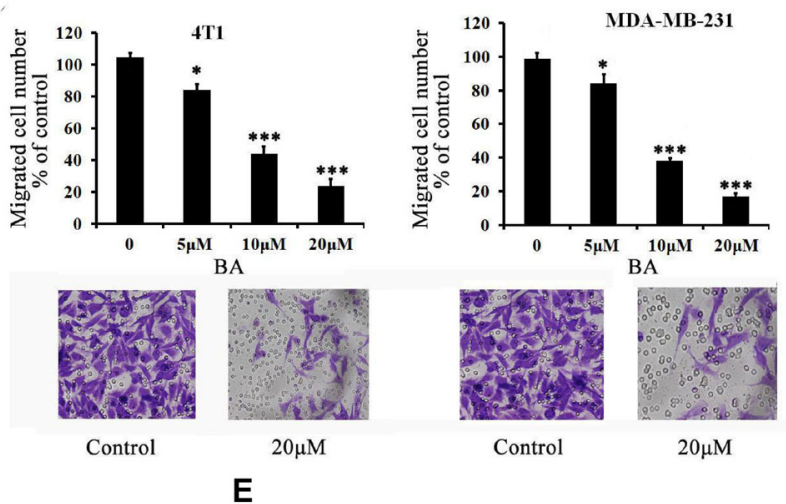

Control

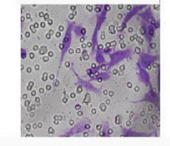

$20 \mu \mathrm{M}$

Figure 2: BA inhibits breast cancer cells 4T1 and MDA-MB-231 migration and invasion. (A) Tumor cells were seeded in six-well plates. We make a 'wound' after the cells grew $\sim 90 \%$ confluence. After incubation for $48 \mathrm{~h}$, the groups were graphed. The black lines indicate the section occupied by the initial scraping, and migrated cells were quantified. (B) Tumor cells were seeded in the roof chamber of transwell with serum-free medium and treated with vehicle or different concentrations of BA. After $48 \mathrm{~h}$, migrated cells were fixed, stained and graphed (20×) and quantified. (C) BA inhibits 4T1 and MDA-MB-231 invasion. Tumor cells were treated with different concentrations of BA and invaded through Matrigel. Invaded cell number was counted $\left({ }^{*} P<0.05 ;{ }^{* *} P<0.01 ;{ }^{* * *} P<0.001\right)$. (D, E) 4T1 and MDA-MB-231 cells were treated with different concentrations of BA. After $48 \mathrm{~h}$, cells were harvested, and western blot assay was performed to detect the expression of MMP-2, MMP-9, TIMP-2, Stat3, P-Stat3, FAK, P-FAK. $\beta$-actin served as loading control. 


\section{Anti-metastasis efficacy of BA in subcutaneous 4T1 model}

The remarkable inhibitory effects of BA on $4 \mathrm{~T} 1$ cells metastasis in vitro implied that it might also efficiently inhibit tumor metastasis in vivo. To verify this hypothesis, we investigated the anti-metastasis activity of $\mathrm{BA}$ in vivo. Firstly, we used a subcutaneous model using 4T1 cells. Then the tumor-bearing mice were treated daily at the dose of $10 \mathrm{mg} / \mathrm{kg}$ for 21 days. There was not different body weight of mice between BA-treated and vehicle-treated groups (Supplementary Figure 4D). However, there was a reduction in tumor growth and tumor weight in the BAtreated groups (Figure 3A, 3B, Supplementary Figure 4A and Supplementary Figure 4C). Moreover, the number of lung metastatic nodules and lungs weight were both decreased after treating by BA (Supplementary Figure 4B, Figure $3 \mathrm{C}$ and $3 \mathrm{D}$ ). In addition, immunohistochemistry analyses were performed to evaluate the anti-metastasis mechanism of BA. As shown in Figure 3E and 3F, BA significantly inhibited the proliferation of nuclear Ki 67 positive cells. Besides, treatment of BA treatment of mice with BA inhibited the expression of MMP-2, MMP-9 and P-Stat3 in 4T1 tumor tissues. Overall, these data suggest that BA could inhibit breast cancer tissues by inhibiting cell proliferation and blocking metastasis,, which is consistent with the results in vitro.

\section{The tumor and lung metastatic environment were inhibited by BA}

MDSCs, as main feature by $\mathrm{CD}_{11 \mathrm{~b}^{+}}$and $\mathrm{Gr}^{+}$ double-positive myeloid cells in mice have been observed. Moreover, the accumulation of MDSCs into the tumors and lungs have an important role in the development of metastasis [30]. Meanwhile, MDSCs are closely related to a lung metastatic in patients with breast cancer [31-33]. Therefore, MDSCs in tumors and lung were quantified by
A

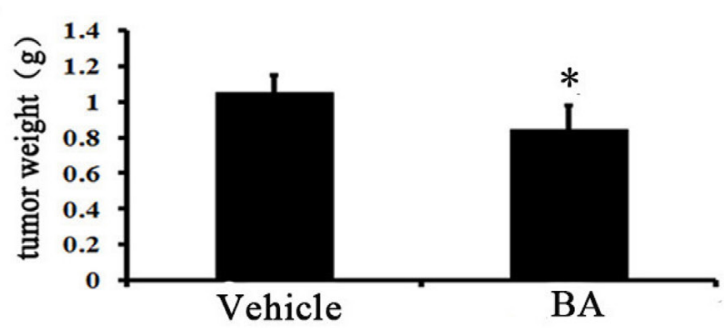

C

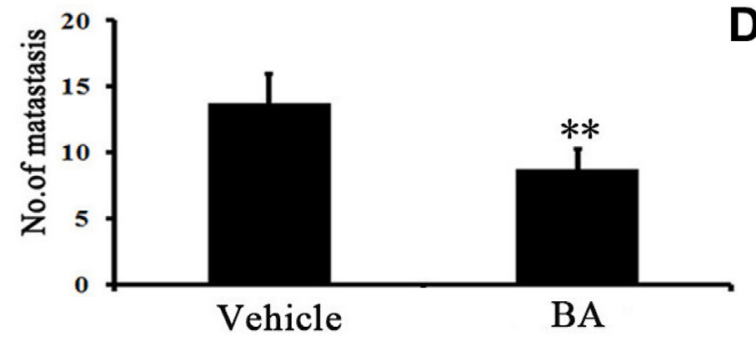

B

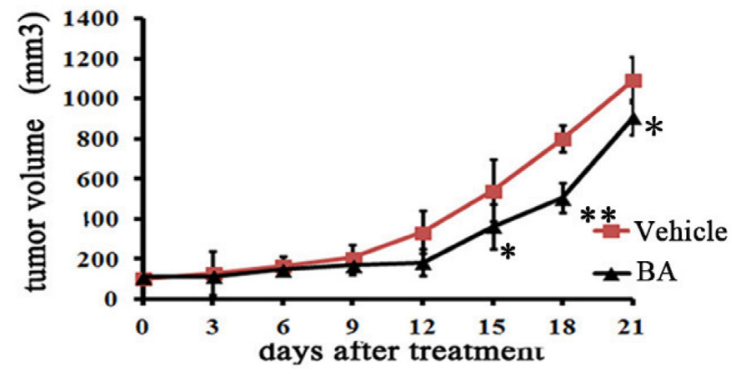

D

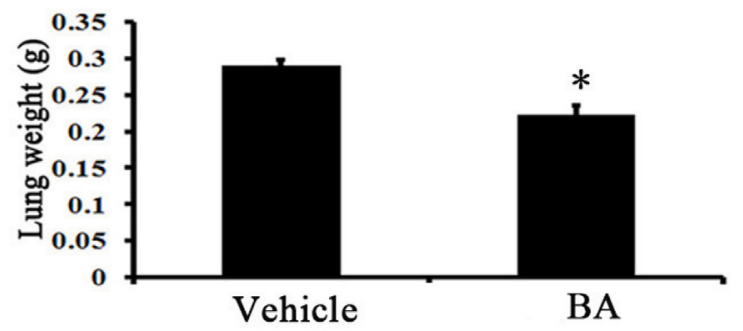

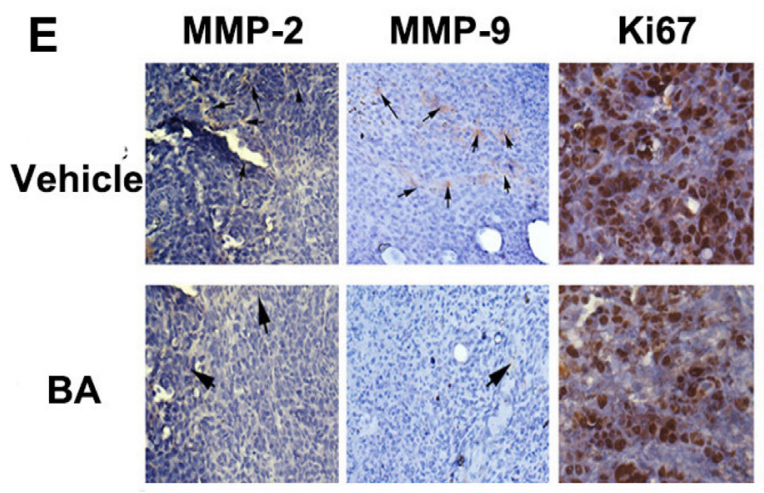
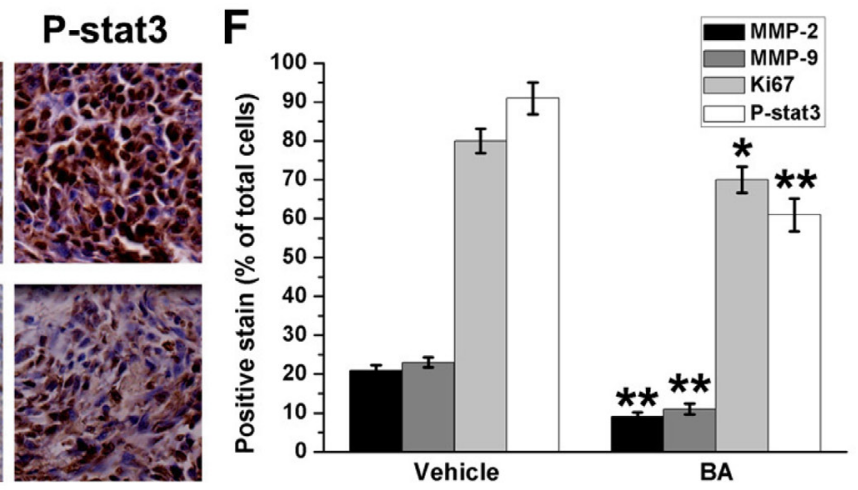

Figure 3: Anti-tumor and anti-metastasis effects of BA in vivo. (A) Represented weight of tumor from mice of different groups (vehicle and BA $(10 \mathrm{mg} / \mathrm{kg} / \mathrm{day}))$, respectively. Data were mean $\pm \mathrm{SD}\left(n=6 ;{ }^{*} P<0.05\right)$. (B) Tumor volume were measured and calculated every three days and presented as mean $\pm \mathrm{SD}\left(n=6 ;{ }^{*} P<0.05,{ }^{* *} P<0.01\right)$. (C) The number of metastasis from lungs. Data were mean $\pm \mathrm{SD}$ $\left(n=6\right.$; $\left.{ }^{*} P<0.05\right)$. (D) The lungs weight of different groups. Results were mean $\pm \operatorname{SD}\left(n=6 ;{ }^{* *} P<0.01\right)$. (E) Immunohistochemistry was performed to measure the expression of MMP-2, MMP-9, Ki67 and P-Stat3 in tumor tissues isolated from vehicle and BA-treated $(10 \mathrm{mg} / \mathrm{kg} / \mathrm{day})$ mice. The treatment with BA markedly reduced MMP-2, MMP-9, Ki67 and P-Stat3-positive cells versus vehicle group (20×). 
FCM analyses in 4T1 tumor-bearing mice after 21 days of treatment. As shown in Figure 4A, the data showed that the percentage of MDSCs in tumor decreased in the $10 \mathrm{mg} / \mathrm{kg}$-treated group compared with the control group. Moreover, we found that $\sim 20 \%$ reduction of MDSCs in the lung (Figure $4 \mathrm{~B}$ ) after $10 \mathrm{mg} / \mathrm{kg}$ of BA treatment. These results suggested that $\mathrm{BA}$ potently reduced the infiltration of MDSCs into the tumors and lung, which could inhibit tumor cell distant colonization.

\section{Safety profile of BA}

As mentioned above, in the BA-treated groups, we did not observed skin ulceration, toxic death and body weight loss (Supplementary Figure 4D) in 4T1 tumorbearing mice. To further elucidate the safety profile of BA, we determined whether the BA could cause the blood system's abnormality. We performed blood chemistry analysis assay and blood routine analysis. Hematological and serum biochemistry analysis of the mice did not show any pathological changes by BA treatment (Figure 5A). Moreover, microscopic examination of the heart, liver, spleen and kidney showed no pathologic changes after treatment with BA (Figure 5B). Furthermore, histological analyses showed that the number of micrometastatic nodules in the BA-treated mice was decreased compared with that of untreated groups.

\section{BA-treated animals have fewer lung metastases}

Previous studies have showed that 4T1 breast tumor have a high metastatic potential and spontaneously metastasize to lung as early as 2 weeks after inoculation [34-36]. To analyze the effects of BA directly in the lungs, 4T1 cells were injected through the tail vein and, 5 days later, mice were treated with BA for 7 days. The lungs were removed and metastatic nodules were quantified. In vehicle-treated groups, multiple large metastasis nodules were evident, whereas the extent of lung metastasis nodules was markedly reduced in BA mice $10 \mathrm{mg} / \mathrm{kg}$ groups (Figure 6A and 6C). Moreover, there was a remarkable decrease in lung weight and numbers of nodules after BA treatment compared with the untreated control (Figure 6B). Overall, these results further indicated that BA could also inhibit the ability of 4T1 cells to grow in the lungs following tail vein injection.

\section{DISCUSSION}

Breast cancer is highly malignant with considerable metastatic potential. Moreover, tumor metastasis possesses a predominant threat to cancer-related mortality and proliferation, migration and invasion of tumor cell are crucial for initial cancer metastasis. [27, 28] Therefore, it is still needed to discover the novel potential drug
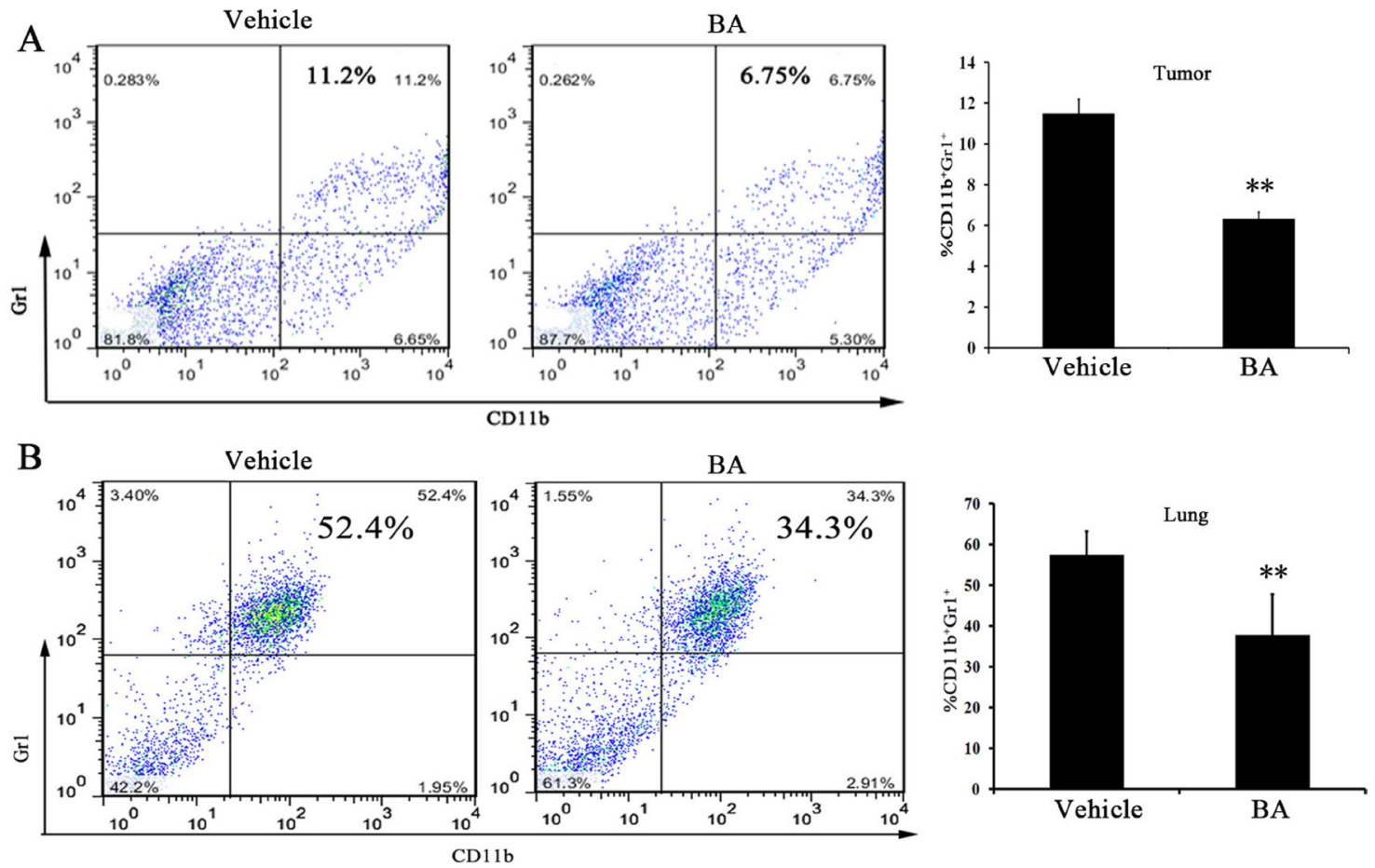

Figure 4: BA reduced lung and tumor $\mathrm{Gr1}^{+} \mathrm{CD} 11 \mathrm{~b}^{+}$MDSCs infiltration. $\mathrm{Gr}^{+}{ }^{+} \mathrm{CD} 11 \mathrm{~b}^{+}$cells were gated and analyzed by FCM for the expression of MDSCs. (A) MDSCs isolated from the tumors of 4T1 tumor-bearing mice in day 21 were treated with vehicle or treated with BA at $10 \mathrm{mg} / \mathrm{kg}$. (B) MDSCs isolated from the lungs of 4T1 tumor-bearing mice in day 21 were treated with vehicle or treated with BA at $10 \mathrm{mg} / \mathrm{kg}$. Values represented mean $\pm \mathrm{SD}\left(n=6 ;{ }^{* *} P<0.01\right)$ 


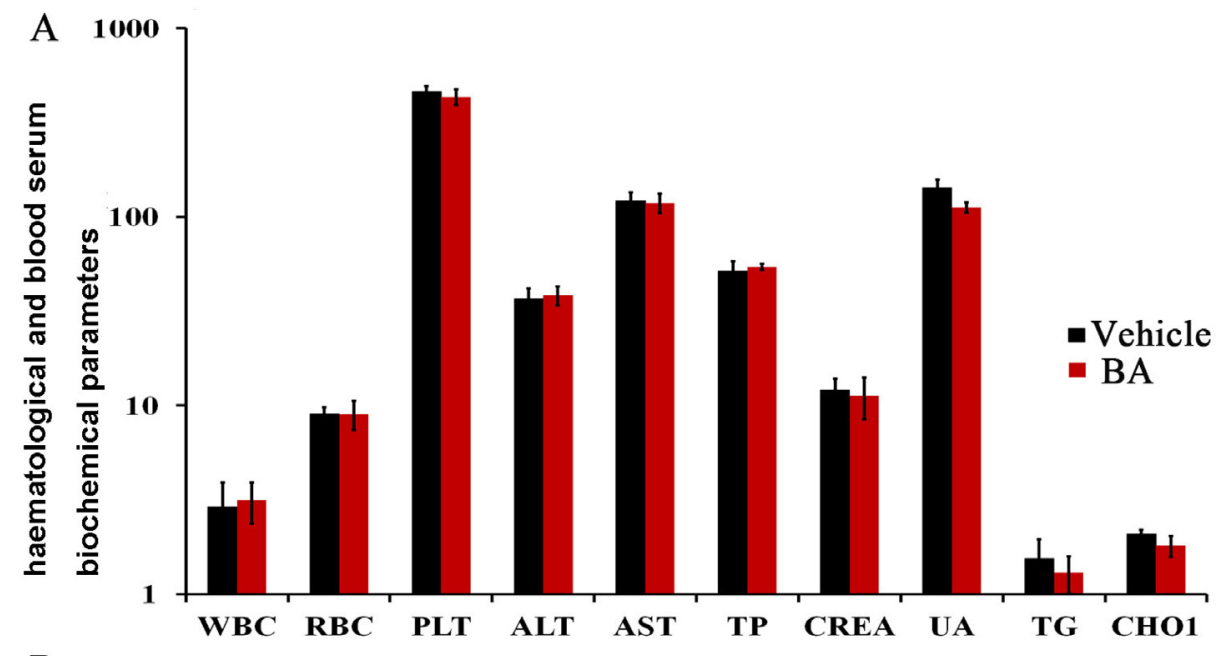

B

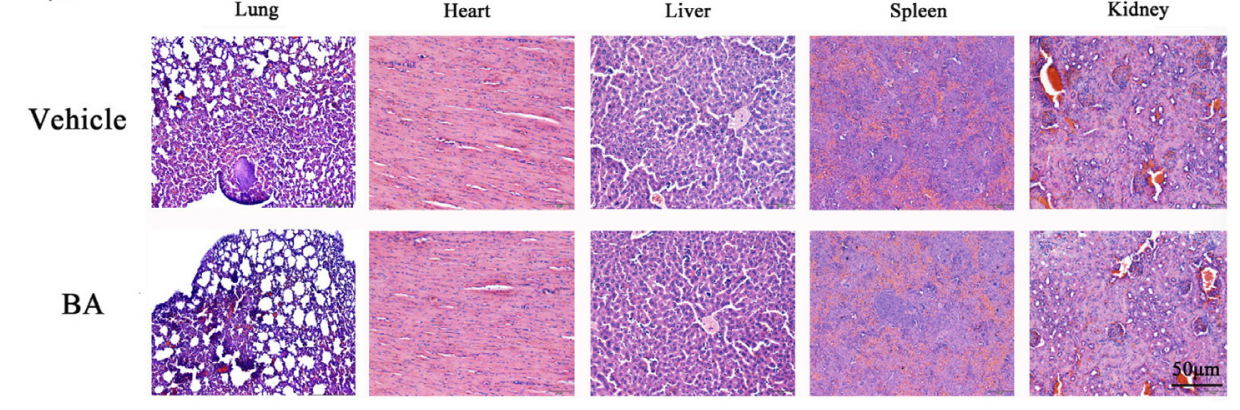

Figure 5: Evaluation of side effects of BA in mice. (A) Hematological and serum biochemistry analysis of blood were done. Units of the parameters are as follows. WBC (white blood cell) and PLT (platelet), 109/1; RBC (red blood cell), 10 12/1; TP (total protein), g/l; ALT (alanine transarninase) and AST (aspartate aminotransferase), U/1; CREA (creatinine) and UA (uric acid), $\mu \mathrm{M} ; \mathrm{TG}$ (triglyceride) and CHOL (cholesterol), mM. (B) BA did not cause obvious pathologic abnormalities in normal tissues. H\&E staining of paraffin-embedded sections of the lung, heart, liver, spleen and kidney (20×).
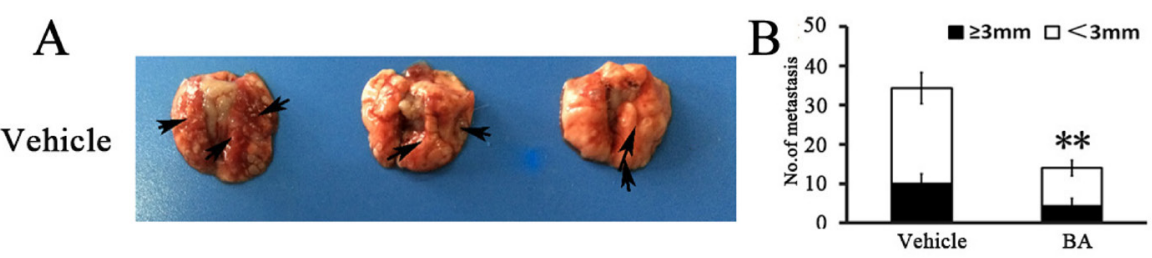

BA
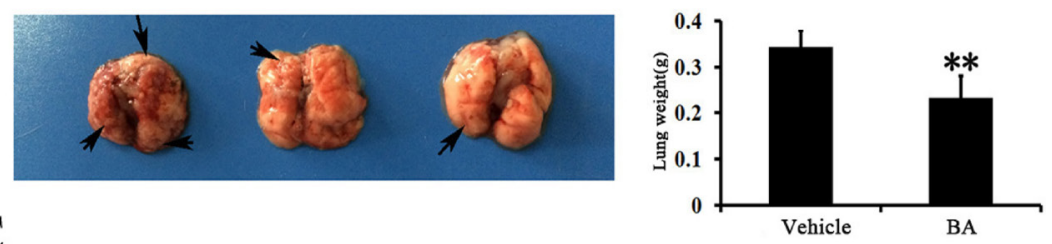

C

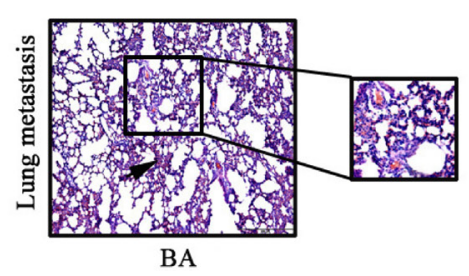

Figure 6: BA inhibited tumor metastasis. (A) Lung metastatic nodules were visualized to show the inhibitory effects of BA on 4T1 intravenous inoculation model in 11 days after treatment. (B) The mean lung metastasis nodules and weight of lungs of each group, the treatment with BA at $10 \mathrm{mg} / \mathrm{kg}$ resulted in significant inhibition of lung metastasis versus vehicle control. Bars showed mean $\pm \operatorname{SD}(n=6$; $\left.{ }^{* *} P<0.01,{ }^{* * *} P<0.001\right)$. (C) Metastases count at day of sacrifice in lung of vehicle and BA-treated groups using H\&E staining. Black arrowheads represented lung metastases. 
candidate to prevent tumor metastasis. In this study, we evaluated whether BA could inhibit the tumor cell migration and invasion for blocking tumor metastasis. Our results showed that BA could inhibit breast cancer cell vitality with low micromole by MTT and clonogenicity assays. From the wound healing migration and invasion assays, our data showed that BA inhibited MDA-MB-231 and 4T1 cell migration and invasion in vitro.

Since the outstanding inhibition effect of BA in proliferation, migration and invasion, we examined the anti-metastasis effects of BA in our established 4T1 tumor model and 4T1 caudal vein model in BALB/c mice. To our delight is that administration of BA at the dose of $10 \mathrm{mg} /$ $\mathrm{kg}$ significantly inhibited breast cancer metastasis to lungs. The results also showed that tumor growth was inhibited by $\mathrm{BA}$ administration $(10 \mathrm{mg} / \mathrm{kg} /$ day $)$. It has been reported that focal adhesion kinase(FAK)/MMP-involved pathway is critical for cancer invasion and metastasis [27]. MMP-2 and MMP-9 activation can particularly enhance tumor cell metastatic potential in breast cancer [37]. On the other hand, MMPs have a positive relationship to invasive behavior of tumors, and tissue inhibitor of MMP-2(TIPM2) which could inhibit activity of MMPs is considered to have inhibitory effect on tumor metastasis [27, 38, 39]. In our study, BA inhibited MMP-2 and MMP9 expression and simultaneously activated TIMP2 in 4T1 and MDA-MB-231 cells. Meanwhile, IHC results showed that BA inhibited the expression of MMP-2 and MMP-9 in vivo. The results suggested that BA could inhibit breast cancer cell invasion and metastasis by blocking MMPs.

Recent studies have also reported that activated Stat3 were closely associated with the development of breast cancer. In addition, constitutive Stat3 also has an important role in controlling cell migration and invasion, suggesting that targeting Stat3 might be a potentially important new form of breast cancer therapy [40]. In the present study, we found that BA not only decreased expression of Stat3 phosphorylation at tyrsione residue 705 but also downregulated P-FAK, MMP-2 and MMP-9 expression in 4T1 and MDA-MB-231 cells. Meanwhile, IHC assay results also showed that BA inhibited the expression of $\mathrm{P}-\mathrm{Stat} 3$ in vivo. These results suggest that BA has significant antitumor and antimetastasis effects, with at least inhibition of Stat3 signaling pathway.

A lot of evidences suggest that the tumor microenvironment can promote tumor development, progression and immune evasion [41, 42]. In particular, MDSCs are important components of the tumor microenvironment. Moreover, MDSCs are present in lots of patients and experimental animals with cancer that decrease immune surveillance and antitumor immunity [43]. In this study, $\mathrm{CD} 11 \mathrm{~b}^{+} / \mathrm{Gr}-1^{+} \mathrm{MDSC}$ in tumors and lungs were quantified by FCM analyses in tumor-bearing mice after BA treatment. Our results indicated that compared with vehicle-treated group, the treatment of mice with BA caused a remarkable decrease in the number of MDSCs in the tumors and lungs. Therefore, it is conceivable that BA can not only potentiate the antitumor effects but also suppress the lung metastasis.Furthermore, our results implied that BA could inhibit lung metastasis in the 4T1 intravenous inoculation model. These results were consistent with the findings in vitro and in vivo, suggesting that breast tumor metastasis inhibited by BA could be mainly ascribed to the impediments of cancer cell migration and invasion.

To sum up, our current project was conducted to investigate the effects of BA on breast cancer metastasis and elucidate its underlying mechanism. As far as we know, this is the first study to demonstrate that BA could block breast cancer cells migration and invasion. Importantly, BA could inhibit lung metastasis by reducing the number of MDSCs in the tumors and lungs. Therefore, these results implied that BA might be a potential therapeutic agent for blocking breast cancer growth and metastasis.

\section{MATERIALS AND METHODS}

\section{Reagents and antibodies}

BA was purchased from (Mansite Chemical Co., Cheng Du, Si chuan, China.). Purity (98.9\%) was measured by high-performance liquid chromatograph(HPLC) analysis. For in vitro assays, BA was prepared initially as a $20 \mathrm{mM}$ stock solution in dimethyl sulfoxide (DMSO) and stored at $-20^{\circ} \mathrm{C}$. Then the stock solution diluted in the relevant assay medium, and $0.1 \%$ DMSO served as a vehicle control. For in vivo studies, BA was prepared in $5 \%$ DMSO in corn oil, and 5\% DMSO in corn oil served as a vehicle control.

MTT and DMSO were bought from Sigma Chemical Co. (St Louis, MO, USA). The primary antibodies against MMP-2, MMP-9, Tissue inhibitor of MMP-2(TIMP-2), cleaved caspase-3 (CC-3), FAK, P-FAK, Stat3, P-Stat3 and $\beta$-actin were purchased from Cell Signaling Technology (Beverly, MA, USA). FITC-CD11b, PE-Gr1 conjugated antibodies were obtained from BD Biosciences (San Diego, CA, USA).

\section{Cell culture}

The human breast cancer cell lines, MCF-7 and MDA-MB-231, the mouse mammary carcinoma cell line 4T1, were obtained from the American Type Culture Collection (Rockville, MD, USA). Cells were propagated in RPMI 1640 or DMEM media containing 10\% heatinactivated fetal bovine serum (FBS; Hyclone, Logan, UT, USA) and $1 \%$ antibiotics (penicillin and streptomycin) in $5 \% \mathrm{CO}_{2}$ at $37^{\circ} \mathrm{C}$.

\section{Cell viability assay}

The cell viability of BA-treated breast cancer cells was assessed by MTT assay. In brief, the exponentially 
growing cells $\left(2 \sim 5 \times 10^{3} /\right.$ well $)$ were plated in $100 \mu \mathrm{l} /$ well in 96-well plates. After $24 \mathrm{~h}$ incubation, the cells were treated with different concentrations of BA. After treatment for 48 and $72 \mathrm{~h}$, respectively, $20 \mu \mathrm{l}$ of $5 \mathrm{mg} / \mathrm{ml}$ MTT was added to each well, and the plates were incubated at $37^{\circ} \mathrm{C}$ for additional $4 \mathrm{~h}$. The medium was subsequently removed, the purple-colored precipitates of formazan were dissolved in $150 \mu \mathrm{l}$ of DMSO. The color absorbance was recorded at 570 or $492 \mathrm{~nm}$ using a Spectra MAX M5 microplate spectrophotometer (Molecular Devices, CA, USA). All experiments were performed in triplicate.

\section{Colony formation assay}

Colony formation assay was measured as previously described [40]. In brief, 4T1, MCF-7 and MDA-MB-231 cells were seeded in specified numbers (300-500 cells/ well) in six-well plates. After $24 \mathrm{~h}$ incubation, the cells were treated with various concentrations of BA and then incubated for additional 12 days. Then the cells were fixed with methanol and stained with a $0.01 \%$ crystal violet solution for $30 \mathrm{~min}$, and the colonies (600 cells) were counted under microscope. Data shown represent the average of three independent experiments.

\section{Western blot analysis}

The western blot analysis was performed as described previously, with minor modification [30]. In brief, 4T1 and MDA-MB-231 cells were treated with BA in designed concentration for $48 \mathrm{~h}$, then, cells were washed twice with cold PBS and lysed in RIPA buffer. The protein concentrations were measured using the Lowry method and equalized before loading. Equal amounts of protein from each sample were subjected to sodium dodecyl sulfate-polyacrylamide gel electrophoresi gels and transferred onto polyvinylidene difluoridemembranes (Amersham Bioscience, Piscataway, NJ, USA). Then, the membranes were blocked for $2 \mathrm{~h}$ at $37^{\circ} \mathrm{C}$ and incubated with specific primary antibodies overnight at $4^{\circ} \mathrm{C}$. After incubation with the relevant secondary antibodies, the reactive bands were identified using an enhanced chemiluminescence kit (Amersham).

\section{Wound-healing migration assay}

Wound-healing migration assay was performed as described previously $[27,30]$. When cancer cells grew to $90 \%$ confluence, cell monolayer was scraped by sterile $0.1 \mathrm{ml}$ pipette tips, and fresh medium which contains only $1 \%$ FBS was added containing different concentrations of BA [44]. After $48 \mathrm{~h}$ incubation, cells were fixed and photographed. Images were acquired using a microscope (Zeiss, Jena, Germany) and the percentage inhibition of migrated cells was expressed using $100 \%$ as the value assigned for untreated group.

\section{Boyden chamber migration and invasion assay}

Boyden chamber ( $8 \mu \mathrm{m}$ pore size) migration assay was performed as previously described, with some modification $[30,45]$. In brief, a total of $1 \times 10^{5}$ cells (for $4 \mathrm{~T} 1$ ) or $5 \times 10^{4}$ cells (for MDA-MB-231) in $100 \mu \mathrm{l}$ serum-free medium were added in the upper chamber, and $600 \mu \mathrm{l}$ of medium containing $10 \%$ FBS was added at the bottom. Different concentrations of BA were added in both chambers. Cells were allowed to migrate for 24 and $48 \mathrm{~h}$. Non-migrated cells in the upper chamber were discarded using a cotton swab. The migrated cells were fixed in methanol and stained with $0.5 \%$ crystal violet. Migrated cells in six randomly selected fields were counted and photographed under a light microscope. Invasion assay was performed according to previous studies [30, 45]. In brief, the upper surface of the transwell membrane were coated with serum-free medium diluted Matrigel (1:3, $60 \mu \mathrm{l} /$ well, BD Biosciences) and the lower compartment of the chambers were filled with $600 \mu \mathrm{l}$ medium with 10 $\%$ FBS. $2 \times 10^{5}$ cells (for $4 \mathrm{~T} 1$ ) or $8 \times 10^{4}$ cells (for MDAMB-231) in $100 \mu \mathrm{l}$ serum-free medium were placed in the upper part of each transwell and treated with different concentrations of BA. After incubation for $48 \mathrm{~h}$, cells on the upper side of the filter were removed. Cells located on the underside of the filter were fixed with methanol and stained with $0.5 \%$ crystal violet, then, migrated cells were counted and photographed under a light microscope. The results were expressed as the percentage inhibition rate of migration compared with untreated group.

\section{Flow cytometry}

Single-cell suspensions from lungs and tumors were prepared as described previously [46]. Then $1 \times 10^{6}$ freshly prepared cells were stained with fluorochrome-conjugated CD11b and Gr1 antibodies. Data were collected by FCM and analyzed with Flow Jo software.

\section{Mice and tumor model}

Female BALB/c mice (6-8 weeks old) were obtained from HFK bioscience CO., LTD, Beijing, China. All animal experiments were approved by the Ethics Committee and Institutional Animal Care and Treatment Committee of Sichuan University (Chengdu, China). In order to verify our results, we used two animal models. One is the subcutaneous immunization model [30]. We inoculated female mice with $4 \mathrm{~T} 1$ cell $(1.0 \times$ $\left.10^{6} / 100 \mu \mathrm{l} / \mathrm{each}\right)$. When visible tumors $\left(\sim 80 \mathrm{~mm}^{3}\right)$ had been developed at the injection sites, the tumor-bearing mice were randomized into two groups (six mice per group), and received intraperitoneally injection of BA $10 \mathrm{mg} / \mathrm{kg}$ or vehicle, respectively, once daily for 21 days. Tumor volumes and body weight were measured every 3 days. The tumor size was calculated according to the formula: Tumor volume $\left(\mathrm{mm}^{3}\right)=0.5 \times \mathrm{L} \times \mathrm{W}^{2}$ where $\mathrm{L}$ 
is the length and $\mathrm{W}$ is the width. At the termination of the experiment, all animals were euthanized by cervical dislocation. At that time tumors and internal organs, such as the heart, livers, spleens, lungs and kidney were excised from animals. Another is intravenous inoculation model. We inoculated female mice with 4T1 cell $\left(2.0 \times 10^{5}\right.$ cells $/ 100 \mu \mathrm{l} /$ mouse $)$. After $\sim 5$ days, the tumorbearing mice were randomized into two groups (six mice per group), and received intraperitoneally injection of BA $10 \mathrm{mg} / \mathrm{kg}$ or vehicle, respectively, once daily for 10 days. At the end point, mice were sacrificed and lungs were dissected.

\section{Immunohistochemistry}

IHC staining of tumor sections were described previously [30]. One part of paraffin tumor sections and lungs sections were stained with hematoxylin and eosin (H\&E). The other part paraffin tumor sections were stained with CC-3, Ki-67, MMP-2 and MMP-9 antibodies. Images were taken with Leica microscope (Leica, DM4000B).

\section{Toxicity evaluation}

To investigate potential side effects or toxicity on mice during the treatment, all the animals were observed continuously for relevant indexes such as body weight, diarrhea, anorexia and other clinical symptoms. On the 21 st day, all animals were euthanized by cervical dislocation after taking blood from eyeball. Blood was obtained for blood routine analysis and blood chemistry analysis. The tissues of heart, liver, spleen, lung and kidney were stained with $\mathrm{H} \& \mathrm{E}$ for histopathologic examination.

\section{Statistical analysis}

All experiments were performed at least in triplicate. Data were represented as mean $\pm \mathrm{SD}$ of three independent experiments. $P$-values for comparison of two groups were determined by two-tailed Student's $t$-test. Statistically significant $P$-values were labeled as follows: ${ }^{*} P<0.05$; ${ }^{* *} P<0.01 ;{ }^{* * *} P<0.001$.

\section{Author contributions}

T.-H. Ye, Y.-W. Zhang, Y.-M. Xie and Y.-Q. Wei designed the experiments. A.-Q. Zeng, T.-H. Ye, Yan Yu, Yuqin Yao, F.-F. Yang and Mengya Liao performed the experiments. A.-Q. Zeng, L.-J. Song, Y.-L. Li, Y. Yu, Y.-J. Li and S.-P. Yang collected and analyzed the data. Y.-L. Deng, P.-Liu, C.-J. Zeng, Y.-Q. Yao and J.-L. Yang provided reagents and advice. A.-Q. Zeng and T.-H. Ye wrote and edited the manuscript.

\section{ACKNOWLEDGMENTS AND FUNDING}

The authors would like to thank Chen Fan for her technical assistance, this study was funded by the grants from the National Natural Science Foundation of China (81500054, 81402564 and 81602950), China Postdoctoral Science Foundation (2015M570788, 2016T90859).

\section{CONFLICTS OF INTEREST}

The authors declare no conflicts of interest.

\section{REFERENCES}

1. Siegel RL, Miller KD, Jemal A. Cancer Statistics, 2015. CA Cancer J Clin. 2015; 65:5-29.

2. Servick K. Breast cancer. Breast cancer: a world of differences. Science. 2014; 343:1452-1453.

3. Chu QD, King T, Hurd T. Triple-negative breast cancer. $\mathrm{I}^{1}$. 2012; 2012:671684

4. Sharpe R, Pearson A, Herrera-Abreu MT, Johnson D, Mackay A, Welti JC, Natrajan R, Reynolds AR, Reis-Filho JS, Ashworth A, Turner NC. FGFR signaling promotes the growth of triple-negative and basal-like breast cancer cell lines both in vitro and in vivo. Clin Cancer Res. 2011; 17:5275-5386.

5. Zhao Z, Lu P, Zhang H, Xu H, Gao N, Li M, Liu C. Nestin positively regulates the $\mathrm{Wnt} /$ beta-catenin pathway and the proliferation, survival and invasiveness of breast cancer stem cells. Breast Cancer Res. 2014; 16:408.

6. Dent RA, Lindeman GJ, Clemons M, Wildiers H, Chan A, McCarthy NJ, Singer CF, Lowe ES, Watkins CL, Carmichael J. Phase I trial of the oral PARP inhibitor olaparib in combination with paclitaxel for first- or secondline treatment of patients with metastatic triple-negative breast cancer. Breast Cancer Res. 2013; 15:R88.

7. Chen S, Han Q, Wang X, Yang M, Zhang Z, Li P, Chen A, Hu C, Li S. IBP-mediated suppression of autophagy promotes growth and metastasis of breast cancer cells via activating mTORC2/Akt/FOXO3a signaling pathway. Cell Death Dis. 2013; 4:e842.

8. Alakurtti S, Makela T, Koskimies S, Yli-Kauhaluoma J. Pharmacological properties of the ubiquitous natural product betulin. Eur J Pharm Sci. 2006; 29:1-13.

9. Fulda S, Kroemer G. Targeting mitochondrial apoptosis by betulinic acid in human cancers. Drug Discov Today. 2009; 14:885-890.

10. Li Y, Li PK, Roberts MJ, Arend RC, Samant RS, Buchsbaum DJ. Multi-targeted therapy of cancer by niclosamide: a new application for an old drug. Cancer Lett. 2014; 349:8-14.

11. Sun YF, Song CK, Viernstein H, Unger F, Liang ZS. Apoptosis of human breast cancer cells induced by 
microencapsulated betulinic acid from sour jujube fruits through the mitochondria transduction pathway. Food Chemistry. 2013; 138:1998-2007.

12. Liu CM, Qi XL, Yang YF, Zhang XD. Betulinic acid inhibits cell proliferation and fibronectin accumulation in rat glomerular mesangial cells cultured under high glucose condition. Biomed Pharmacother. 2016; 80:338-342.

13. Luo R, Fang D, Chu P, Wu H, Zhang Z, Tang Z. Multiple molecular targets in breast cancer therapy by ${ }^{2}$. Biomed Pharmacother. 2016; 84:1321-1330.

14. Fulda S. Betulinic acid: a natural product with anticancer activity. Mol Nutr Food Res. 2009; 53:140-146.

15. Gheorgheosu D, Duicu O, Dehelean C, Soica C, Muntean D. Betulinic acid as a potent and complex antitumor phytochemical: a minireview. Anti-Cancer Agent Me. 2014; 14:936-945.

16. Hsu TI, Chen YJ, Hung CY, Wang YC, Lin SJ, Su WC, Lai MD, Kim SY, Wang Q, Qian K, Goto M, Zhao Y, Kashiwada Y, et al. A novel derivative of betulinic acid, SYK023, suppresses lung cancer growth and malignancy. Oncotarget. 2015; 6:13671-13687. https://doi.org/10.18632/ oncotarget. 3701 .

17. Hsu RJ, Hsu YC, Che SP, Fu CL, Yu JC, Chang FW, Chen YH, Liu JM, Ho JY, Yu CP. The triterpenoids of Hibiscus syriacus induce apoptosis and inhibit cell migration in breast cancer cells. BMC Complement Altern Med. 2015; 15:65.

18. Hsu TI, Wang MC, Chen SY, Huang ST, Yeh YM, Su WC, Chang WC, Hung JJ. ${ }^{3}$ the sumoylation of sp1 to inhibit lung cancer growth. Mol Pharmacol. 2012; 82:1115-1128.

19. Chintharlapalli S, Papineni S, Lei P, Pathi S, Safe S. Betulinic acid inhibits colon cancer cell and tumor growth and induces proteasome-dependent and-independent downregulation of specificity proteins $(\mathrm{Sp})$ transcription factors. BMC cancer. 2011; 11:371.

20. Li L, Du Y, Kong X, Li Z, Jia Z, Cui J, Gao J, Wang G, Xie $\mathrm{K}$. Lamin B1 is a novel therapeutic target of betulinic acid in pancreatic cancer. Clin Cancer Res. 2013; 19:4651-4661.

21. Reiner T, Parrondo R, de Las Pozas A, Palenzuela D, Perez-Stable C. Betulinic acid selectively increases protein degradation and enhances prostate cancerspecific apoptosis: possible role for inhibition of deubiquitinase activity. PLoS One. 2013; 8:e56234.

22. Damle AA, Pawar YP, Narkar AA. Anticancer activity of betulinic acid on MCF-7 tumors in nude mice. Indian J Exp Biol. 2013; 51:485-491.

23. Bar FM, Khanfar MA, Elnagar AY, Liu H, Zaghloul AM, Badria FA, Sylvester PW, Ahmad KF, Raisch KP, El Sayed KA. Rational design and semisynthesis of betulinic acid analogues as potent topoisomerase inhibitors. J Nat Prod. 2009; 72:1643-1650.

24. Parrondo R, de las Pozas A, Reiner T, Rai P, Perez-Stable C. NF-kappaB activation enhances cell death by antimitotic drugs in human prostate cancer cells. Mol Cancer. 2010; 9:182.

25. Ng KW, Salhimi SM, Majid AM, Chan KL. Anti-angiogenic and cytotoxicity studies of some medicinal plants. Planta Med. 2010; 76: 935-940.

26. Chen JJ, Patel A, Sodani K, Xiao ZJ, Tiwari AK, Zhang DM, Li YJ, Yang DH, Ye WC, Chen SD, Chen ZS. bba, a synthetic derivative of 23- hydroxybutulinic acid, reverses multidrug resistance by inhibiting the efflux activity of MRP7 (ABCC10). PLoS One. 2013; 8:e74573.

27. Zhang T, Li J, Dong Y, Zhai D, Lai L, Dai F, Deng H, Chen Y, Liu M, Yi Z. Cucurbitacin E inhibits breast tumor metastasis by suppressing cell migration and invasion. Breast Cancer Res Treat. 2012; 135:445-458.

28. Weigelt B, Peterse JL, van't Veer LJ. Breast cancer metastasis: markers and models. Nature reviews. Cancer. 2005; 5:591-602.

29. Kessenbrock K, Plaks V, Werb Z. Matrix metalloproteinases: regulators of the tumor microenvironment. Cell. 2010; 141:52-67.

30. Ye TH, Wei XW, Yin T, Xia Y, Li DL, Shao B, Song XJ, He SS, Luo M, Gao X, He ZY, Luo C, Xiong Y, et al. Inhibition of FGFR signaling by PD173074 improves antitumor immunity and impairs breast cancer metastasis. Breast Cancer Res Treat. 2014; 143:435-446.

31. Kodumudi KN, Woan K, Gilvary DL, Sahakian E, Wei S, Djeu JY. A novel chemoimmunomodulating property of docetaxel: suppression of myeloid-derived suppressor cells in tumor bearers. Clin Cancer Res. 2010; 16:4583-4594.

32. Gabrilovich DI, Nagaraj S. Myeloid-derived suppressor cells as regulators of the immune system. Nat Rev Immunol. 2009; 9:162-174.

33. Xin H, Herrmann A, Reckamp K, Zhang W, Pal S, Hedvat M, Zhang C, Liang W, Scuto A, Weng S, Morosini D, Cao $\mathrm{ZA}$, Zinda $\mathrm{M}$, et al. Antiangiogenic and antimetastatic activity of JAK inhibitor AZD1480. Cancer Res. 2011; 71:6601-6610.

34. Pulaski BA, Terman DS, Khan S, Muller E, OstrandRosenberg S. Cooperativity of Staphylococcal aureus enterotoxin B superantigen, major histocompatibility complex class II, and CD80 for immunotherapy of advanced spontaneous metastases in a clinically relevant postoperative mouse breast cancer model. Cancer Res. 2000; 60:2710-2715.

35. Wang YS, Li D, Shi HS, Wen YJ, Yang L, Xu N, Chen XC, Chen X, Chen P, Li J, Deng HX, Wang CT, Xie G, et al. Intratumoral expression of mature human neutrophil peptide-1 mediates antitumor immunity in mice. Clin Cancer Res. 2009; 15:6901-6911.

36. Pulaski BA, Ostrand-Rosenberg S. Reduction of established spontaneous mammary carcinoma metastases following immunotherapy with major histocompatibility complex 
class II and B7.1 cell-based tumor vaccines. Cancer Res. 1998; 58:1486-1493.

37. Liotta LA, Tryggvason K, Garbisa S, Hart I, Foltz CM, Shafie S. Metastatic potential correlates with enzymatic degradation of basement membrane collagen. Nature. 1980; 284:67-68.

38. Curran S, Murray GI. Matrix metalloproteinases in tumour invasion and metastasis. J Pathol. 1999; 189:300-308.

39. Karavasilis V, Malamou-Mitsi V, Briasoulis E, Tsanou E, Kitsou E, Kalofonos H, Fountzilas G, Fotsis T, Pavlidis N. Matrix metalloproteinases in carcinoma of unknown primary. Cancer. 2005; 104:2282-2287.

40. Yang FF, Hu MX, Lei Q, Xia Y, Zhu YX, Song XJ, Li Y, Jie H, Liu CQ, Xiong Y, Zuo ZP, Zeng AQ, Li YL, et al. Nifuroxazide induces apoptosis and impairs pulmonary metastasis in breast cancer model. Cell Death Dis. 2015; 6:e1701

41. Kortylewski M, Swiderski P, Herrmann A, Wang L, Kowolik C, Kujawski M, Lee H, Scuto A, Liu Y, Yang C, Deng J, Soifer HS, Raubitschek A, et al. In vivo delivery of siRNA to immune cells by conjugation to a TLR9 agonist enhances antitumor immune responses. Nat Biotech. 2009; 27:925-932.

42. Place AE, Jin Huh S, Polyak K. The microenvironment in breast cancer progression: biology and implications for treatment. Breast Cancer Res. 2011; 13:227.

43. Nagaraj S, Gabrilovich DI. Myeloid-derived suppressor cells in human cancer. Cancer J. 2010; 16:348-353.

44. Saadoun S, Papadopoulos MC, Hara-Chikuma M, Verkman AS. Impairment of angiogenesis and cell migration by targeted aquaporin-1 gene disruption. Nature. 2005; 434:786-792.

45. Cao H, Zhang J, Liu H, Wan L, Zhang H, Huang Q, Xu E, Lai M. IL-13/STAT6 signaling plays a critical role in the epithelial-mesenchymal transition of colorectal cancer cells. Oncotarget. 2016; 7:61183-61198. https://doi.org/10.18632/ oncotarget. 11282.

46. Kortylewski M, Kujawski M, Wang T, Wei S, Zhang S, Pilon-Thomas S, Niu G, Kay H, Mule J, Kerr WG, Jove $\mathrm{R}$, Pardoll D, Yu H. Inhibiting Stat3 signaling in the hematopoietic system elicits multicomponent antitumor immunity. Nat Med. 2005; 11:1314-1321. 